\title{
Iron and chromium doped titanium dioxide nanotubes for the degradation of environmental and industrial pollutants
}

\author{
*S. T. Hussain; A. Siddiqa \\ Nano Science and Catalysis Division, National Center for Physics, Quaid-i-Azam University Complex, Islamabad, \\ Pakistan \\ Received 25 June 2010; $\quad$ revised 12 October 2010; $\quad$ accepted 12 February 2011; $\quad$ available online 1 March 2011

\begin{abstract}
Pure titanate nanotubes and titanate nanotubes doped with iron (III) and chromium (III) were fabricated by the hydrothermal treatment in methanol and sodium hydroxide mixture. The fabricated nano tubes have high surface area, high aspect ratio, consisted of very good surface morphology and high metals dispersion. The morphology, crysralline phase, composition were characterized by powdered X-ray diffraction, scanning electron microscopy, transmission electron microscopy, Barrett-Joyner-Halenda methods and X-ray photoelectron spectroscopy. The results showed that nanotubes possess anatase phase and are composed up of 8-12 nm in diameter and 360-400 $\mathrm{nm}$ in length. The band gap of the titanium dioxide nanotubes was determined using transformed diffuse reflectance spectroscopy according to the Kubelka-Munk theory, showed pronounced band gap decrease on doped titanium dioxide nanotubes. The photocatalytic activity of doped nanotubes were evaluated in terms of degradation of phenol and photoreduction of carbon dioxide into methanol and ethanol under Ultra violet and Infra red irradiation. It was found that with iron (III) and chromium (III) doped titanium dioxide nanotubes exhibited much higher photocatalytic activity than undoped titanate nanotubes.
\end{abstract}

Keywords: Titanium dioxide; Hydrothermal method; Infra red;Phenol degradation; Photocatalyst; Sun irradiation

\section{INTRODUCTION}

Following the discovery of nanotube morphology in carbon, many studies have been devoted to the preparation and characterization of various inorganic nanotubular materials (Mohapatra et al., 2007; Goyal et al., 2008). Among these nanostructures, the titanium dioxide nanotubalar materials are of high interest due to chemical inertness, strong oxidizing power, large surface area, non toxicity, high photocatalytic activitry, high cation exchange capacity, strong oxidizer, low cost of production and relatively good stability at elevated temperatures (Khan et al., 2006; Samarghandi et al., 2007; Liang and Li, 2009). This material extensively explored as a catalyst for water splitting and for the production of solar hydrogen (Chen et al., 2005). As a catalyst for the conversion of green house gases into energy producing products for methane and methanol (Chen et al., 2005; Khan et al., 2006). Moreover these nanotubes are widely exploited in lithium ions batteries, electrochemical devices, gas sensors, photoluminescence ion exchange and in

*Corresponding Author Email: dr_tajammul@yahoo.ca Tel.: +9251 2077 308; Fax: +9251 2077395 photovoltaic dye sensitized solar cells (Kuang et al., 2008).

Recent research on Titanium dioxide $\left(\mathrm{TiO}_{2}\right)$ nanotubes is mainly focused on the understanding of its strong photocatalytic activity which is useful in environmental pollution remediations, such as air purification, hazardous waste remediation and water purification (Qamar, 2008; Sekabira et al., 2010). The presence of pollutants $\mathrm{CO}_{2}, \mathrm{NO}_{2}$ in air, organic dyes (methylene blue, reactive blue, methyl red etc) and many other organic hydrocarbons (phenol, formaldehyde and gasoline), produced as a result of many industrial processes (manufacture of dyes, food processing, pesticides, polymers) in industrial wastes, have caused the sever environmental problems (Chein and shih, 2007; Ismael et al., 2007; Qamar, 2008). Titanium dioxide material has sufficient band gap energies for specifically promoting or catalyzing a wide range of chemical reactions of environmental interest (Ismael et al., 2007). In particular, it has been used to oxidize industrial pollutants and its conversion into products like of $\mathrm{H}_{2} \mathrm{O}$, alcohols and other useful hydrocarbons. 
However, powdered photocatalyst have limitations. It needs post treatment separation in a slurry system after photocatalyst reaction. Though this can be overcome by converting $\mathrm{TiO}_{2}$ into nanotubes which resulted in increase surface area and catalytic activity (Fujishima et al., 2006; Khanfekr et al., 2009; Zhu et al., 2009; Rafati et al.,2010). Interest in $\mathrm{TiO}_{2}$ nanotubes photocatalysis increased after the pioneering study of Fujishma and Honda in 1972. Kochkar et al. (2009) discovered the photocatalytic splitting of water on $\mathrm{TiO}_{2}$ electrodes. Furthermore, Coating the nanotubes with transition metals resulted in boosting of their activity (Dmitry et al., 2005; Kochkar et al., 2009).

$\mathrm{TiO}_{2}$ nanotubes were reported first time by Hoyer via template assisted method. Latter on $\mathrm{TiO}_{2}$ based nanotubes (TNTs) of different geometrical shapes and microstructures have been developed through various approaches, for example the electrochemical anodic oxidation of Ti, assisted tempelate method and alkaline hydrothermal treatment (Wang et al., 2004; Khan et al., 2006; Mohapatra et al., 2007; Liang and Li 2009). There are advantages and limitations in each of above mentioned methods like attaining uniform inner diameter of titanium dioxide nanotubes and controlling the morphology, shape and length of the synthesized nanotubes (Wang et al., 2004; Liang and Li 2009).

The most widely usedtemplate-assisted method which utilizes porous alumina, carbon nanotubes or polymer membrane and amphiphilic surfactant as template to construct material with a regular morphology (Macak et al., 2007) also has the disadvantage in pre-fabrication and removal of template and usually results in impurities encapsulation. The highly ordered and self assembled $\mathrm{TiO}_{2}$ nanotubes were discovered by Grimes in 2001 via anodization of Ti foil in fluoride based electrolyte. This method has limitations because it results in low yield and involvement of highly expense fabrication apparatus (Wang et al., 2004; Macak et al., 2007).

Hydrothermal method was reported first time by Kasuga et al. (1998) for preparation of $\mathrm{TiO}_{2}$ nanotubes in one step without use of template, by treating $\mathrm{TiO}_{2}$ with concentrated $\mathrm{NaOH}$ at elevated temperature in a tightly closed vessel.

Titanium dioxide nanotube produced by this method have good uniformity, controllable size, large surface area, high reactivity and in the amorphous state (Yury et al., 2000; Liang and Li 2009). The hydrothermal process too has problems for its extensive production, due to low yield. This method could also not produce well separated and crystallized nanotubes.

The mechanism of formation and the morphology (diameter and length, wall thickness, size distribution and size of nanotube agglomerates) of nanotubes are still an issue of debate (Yury et al., 2000; Chen et al., 2002a; Sun and Li 2003). Hydrothermal methods of TNTs formation using $\mathrm{TiO}_{2}$ as raw material (in the range of temperature between $110^{\circ} \mathrm{C}-180^{\circ} \mathrm{C}$ and time of $24-96 \mathrm{~h}$ ) under high molar concentration of aqueous $\mathrm{NaOH}$ $(10 \mathrm{M})$, has been reported earlier. In the present study, the limitation of long reaction time, high temperature, and highly alkali concentration have been overcome using $\mathrm{NaOH}$ and methanol mixture rather than water. This resulted in nanotubes formation at low temperature $\left(90^{\circ} \mathrm{C}\right)$, reaction time $(8 \mathrm{~h})$ and at stirring rate of $300 \mathrm{rpm}$

In the present study a new synthesis procedure for the production of $\mathrm{Fe} / \mathrm{Cr}$ doped and undoped $\mathrm{TiO} 2$ nanotubes via hydrothermal synthesis from $\mathrm{TiO}_{2}$ nanoparticles and its application for the degradation of organic waste, like phenol and carbon dioxide conversion to alcohol using UV radiation, is reported. The effect of dopant on degradation of phenol, conversion of carbon dioxide to alcohols and on its optical properties were also studied, the results compared with other studies described above. The data suggest the improvement not only in the synthesis, but also in its industrial applications. The study was carried out in January-March , 2010 at the Nanoscience Catalysis Division, National Centre for Physics, Quaidi-Azam University, Islamabad, Pakistan.

\section{MATERIALS AND METHODS}

\section{Reagents}

Titanium dioxide, anatase, sodium hydroxide $(\mathrm{NaOH})$, Methanol, Hydrochloric acid $(\mathrm{HCl})$, phenol, $\mathrm{FeCl}_{3}$ and $\mathrm{CrCl}_{3}$ were obtained from Aldrich and were used without further purification. Ultra pure water was used without purification in the whole experiment.

\section{Preparation of Titanium dioxide nanotubes}

Titanium dioxide nanotubes were produced using hydrothermal method similar to that described in literature (Yury et al., 2000) with the modification of using methanol than water. In typical nanotube preparation, $3 \mathrm{~g}$ of the $\mathrm{TiO}_{2}$ powder was mixed with 300 $\mathrm{mL}$ of $2 \mathrm{M} \mathrm{NaOH}$ in methanol, followed by hydrothermal treatment in a Teflon-lined autoclave high-pressure 
stainless steel at $90{ }^{\circ} \mathrm{C}$ for $8 \mathrm{~h}$ and at stirring speed of $300 \mathrm{rpm}$. After hydrothermal treatment the precipitates were separated through filteration and washed with $0.1 \mathrm{M}$ $\mathrm{HCl}$ and distilled water numerous times until the $\mathrm{pH}$ of the filterate turned to 7 . The resulting sample was dried in oven at $100^{\circ} \mathrm{C}$ overnight to get TNTs (titanium dioxide nanotubes) and labelled as A-TNTs for undoped titanium dioxide nanotubes.

\section{Prepration of Fe/Cr doped Titanium nanotubes}

$\mathrm{Fe} / \mathrm{Cr}$-codoped $\mathrm{TiO}_{2}$ nanotubes were prepared as follows: The synthesized titanium dioxide nanotube (ATNTs), $1 \mathrm{~g}$, was stirred with $100 \mathrm{~mL}$ aqueous solutions containing $0.1 \mathrm{~g}$ each of $\mathrm{FeCl}_{3}, \mathrm{CrCl}_{3}$ for $10 \mathrm{~min}$. The solution was transferred into a stainless steel autoclave with a Teflon lined autoclave, which was sealed and maintained at $90^{\circ} \mathrm{C}$ for $2 \mathrm{~h}$ under $\mathrm{H}_{2}$-pressure of 25 bar to dope titanium dioxide nanotubes by ion exchange treatment. The $\mathrm{H}^{+}$ion existing within nanotubes was ion exchanged with above referred metal ions. Subsequently, the resultant materials were centrifuged, washed and dried at $60^{\circ} \mathrm{C}$ for $4 \mathrm{~h}$ to get modified titanium dioxide nanotubes. These samples were labelled as BTNTs for Fe/Cr co-doped titanium dioxide nanotubes.

\section{Photocatalytic activity}

The degradation of phenol and conversion of $\mathrm{CO}_{2}$ were investigated in photocatalytic batch reactor setup as shown in Fig. 1a and b. In two separate experiments $0.5 \mathrm{~g}$ of A-TNTs and B-TNTs were added in $0.01 \mathrm{M}$ phenol for phenol degradation while for conversion of $\mathrm{CO}_{2}$, $0.2 \mathrm{~g}$ of A-TNTs and B-TNTs were suspended into $0.2 \mathrm{~N}$
$\mathrm{NaOH}$ solution with pure $\mathrm{CO}_{2}$ bubbling at the rate of $100 \mathrm{mls} / \mathrm{min}$. In both systems a mercury lamp (UltraViolet Products Inc., USA; LF-204.LS), UVC (254 nm) was placed on the top of reactor. The reaction was carried out for 1-2 $\mathrm{h}$ with vigorous stirring (300 rpm) at room temperature. After completion of reaction TNTs were separated through filteration using milipore filter paper (with the pore size of $125 \mathrm{~mm}$ ). The filterate analyzed using GC/MS after the chloroform extraction method.

\section{Characterization}

The microstructures and morphology of the ATNTs and B-TNTs were observed using Transmission Electron Microscope S-3500 N with Absorbed Electron Detector S-6542 (Hitachi Science System Ltd) and SEM scanning electron microscopy with energy dispersive X-ray spectrometer (SEM-EDX, Philips XL30, EDAX DX4). XRD patterns were recorded using a Scintag XDS $2000 \mathrm{D}_{8}$ diffractometer equipped with $\mathrm{CuK} \alpha$ radiation of wavelength of $1.5406 \mathrm{~A}^{\circ}$ in the $2 \theta$ range $20^{\circ}-80^{\circ}$ with a step size of $0.02^{\circ}$ to evaluate the crystallinity, phase and structure. Brunauer Emmett Teller (BET) measurements were done to determine specific surface area nitrogen adsorption-desorption and average pore volume was also evaluated from the data using the Barret-JoynerHalender (BJH) method. EDX (Inca Energy, Oxford Instrument Microanalysis, Ltd) were used to determine the surface comparison of samples under specified conditions of $20 \mathrm{keV}, 25 \mathrm{~mm}$ working distance and magnification varying from x 300-1000.
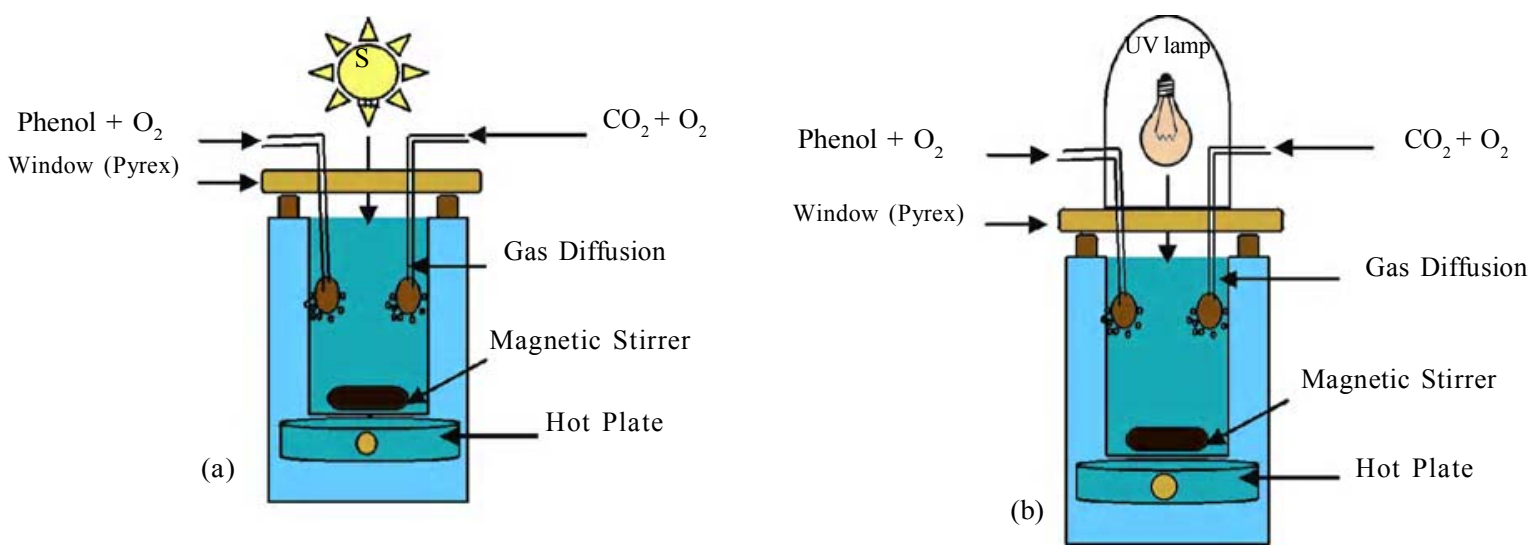

Fig. 1 ( $\mathrm{a}$ and $\mathrm{b}$ ): Illustration of batch type reactor for Phenol and $\mathrm{CO}_{2}$ reaction 
Diffuse reflectance spectroscopy (DRS) was used for the optical characterization of $\mathrm{TiO}_{2}$ nanotubes. All spectra were taken at room temperature in the range of 200-1000 nm on Lambda-950 Perkin-Elmer.

The chemical status and shift of the catalyst surface due to doping were analyzed by X-ray photoelectron spectroscopy (XPS) using a VG Microtech MT500 spectrometer, operated with a constant pass energy of $50 \mathrm{eV}$ and with $\mathrm{Mg}$ Ká radiation as the excitation source ( $h i=1253.6 \mathrm{eV}$ ). The catalyst was pressed into a pellet, and then adhered on sample holder by carbon tape.

\section{RESULTS AND DISCUSSIONS}

The use of $\mathrm{H}_{2} \mathrm{O}$ in the previous study results in the formation of rutile/anatase nanotubes mixture with low yield. The staring $\mathrm{TiO}_{2}$ nanoparticles with mixed phases on anatase and rutile could be separated well into $\mathrm{TiO}_{6}$ octahedra in the $\mathrm{NaOH}$ basic solution and methanol which has boiling point of $64.7^{\circ} \mathrm{C}$ as compared to $\mathrm{H}_{2} \mathrm{O}$. This is reason that in our process nanotubes are formed at much lower temperature, lower pressure and in considerably less time. For the rearrangement of $\mathrm{TiO}_{6}$ octahedra, methanol molecules play the role of bridges between surface $\mathrm{OH}$ groups of different octahedral which share only one common yertex, using the two lone pairs of electrons on the oxygen. The deprotonated methylate produced from methanol can chelate $\mathrm{TiO}_{6}$, that is beneficial to the edge-sharing polycondesation between $\mathrm{TiO}_{6}$ octahedra to form anatase phase rather than rutile phase nanotubes in the hydrothermal conditions.

The phase and crystal structure of TNTs before and after calcinations in air at $450{ }^{\circ} \mathrm{C}$ for $2 \mathrm{~h}$ were investigated by XRD pattern and shown in Fig. 2a and $\mathrm{b}$ for undoped and doped TNTs. The intensities and positions of the observed peaks are in agreement with literature values (Zhuang et al., 2007). The XRD peaks were sharp which suggests that synthesized nanotubes have relatively high crystallinity and attributable to the anatase phase (indicated by peak 24 ). The peaks became sharper and broader after doping which demonstrates that cryatallanity of nanotubes enhances with the doping.

BET specific surface area of doped and undoped TNTs is much higher to the $\mathrm{TiO}_{2}$ nanoparticles. BET surface area has no effect on the phase of the TNTs but has an effect on the photcatalytic degradation reaction (Hussain et al., 2009). Doping on titanium dioxide nanotubes with metals resulted in increase in BET surface area and pore volume. Larger surface area increased adsorption/ desorption capacity and consequently good photocatalytic activity (Diebold, 2002; Hussain et al., 2009). Table 1 presents the measured surface area of the prepared titanium dioxide nanotubes; the surface area of the commercial $\mathrm{TiO}_{2}$ is also included for comparison. Fig. 3a and b and Fig. 4a and $b$ shows the SEM and TEM image of doped and undoped TNTs. As seen in the SEM image, $\mathrm{TiO}_{2}$ nanotubes have had obvious tubular and uniform morpholgy with an average diameter of 8-12 nm and with narrow size distribution.. The dispersion of $\mathrm{Fe} / \mathrm{Cr}$ on the nanotubes is uniform as depicted in Fig. $3 \mathrm{~b}$.

TEM analysis verified that tubes were hollow with an open end having needle shape structures. Furthermore, TEM pattern shows that these tubes

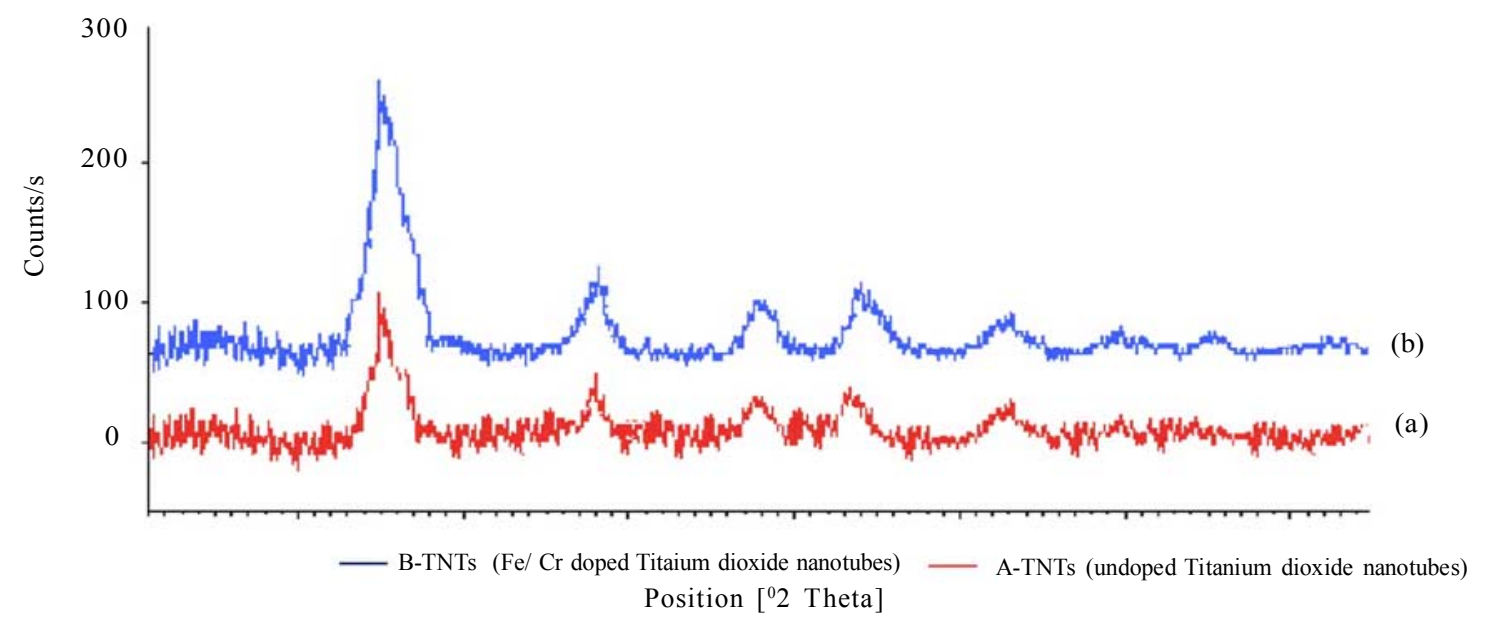

Fig. 2: XRD patterns of (a) A-TNTs (undoped Titanium dioxide nanotubes) (b) B-TNTs (Fe/ Cr doped Titaium dioxide nanotubes) 
are uniform, single walled and are upto several hundred $\mathrm{nm}$ in length. No $\mathrm{TiO}_{2}$ nanoparticles exist around the nanotubes, proving high conversion of nanoparticles into nanotubes under employed experimental conditions. Moreover, the chemical composition and respective percentage of each atom present in the samples, was analyzed by EDX. Table 2 presents the percentages of $\mathrm{O}, \mathrm{Ti}, \mathrm{Na}, \mathrm{Fe}$ and $\mathrm{Cr}$ elements confirming the formation of doped and undoped TNTs.

\section{The DRS studies}

The band gap of samples was measured by KubelkaMunk function $F(R)$ which is related to the the diffuse reflectance, $\mathrm{R}$, of the sample according to the following relation (Diebold 2002; Hussain et al., 2009).

Table 1: BET surface area and pore volume of doped and undoped samples and commercial Degussa P25

\begin{tabular}{lccc}
\hline Samples & A-TNTs & B-TNTs & Commercial (Degussa) \\
\hline Surface area $\left(\mathrm{m}^{2} / \mathrm{g}\right)$ & 365 & 400 & 64 \\
Pore volume $/\left(\mathrm{cm}^{3} / \mathrm{g}\right)$ & 0.611 & 0.707 & 0.09 \\
\hline
\end{tabular}

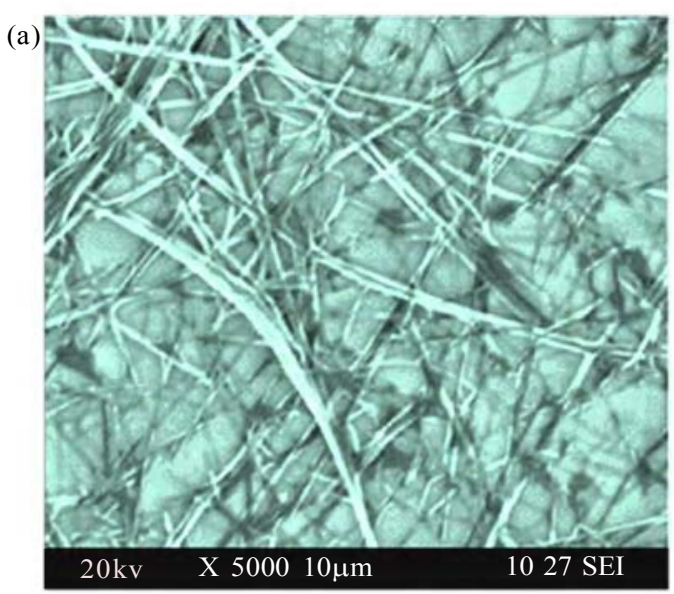

Fig. 3: SEM images of

(a) A-TNTs

(b) B-TNTs

(a)

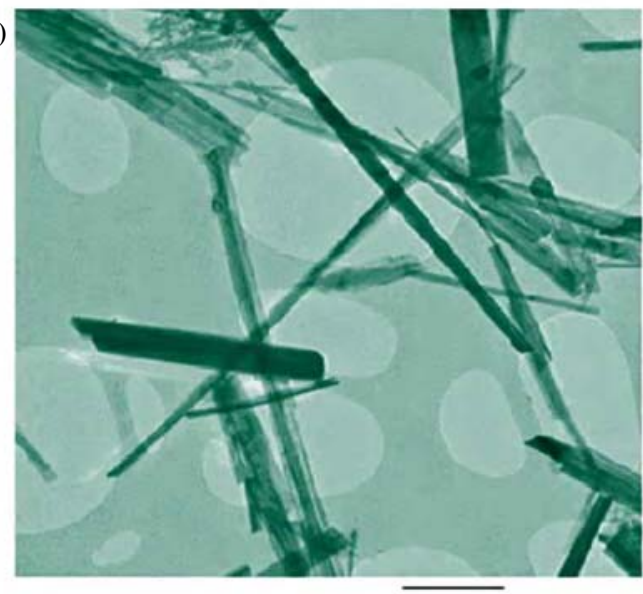

(b)

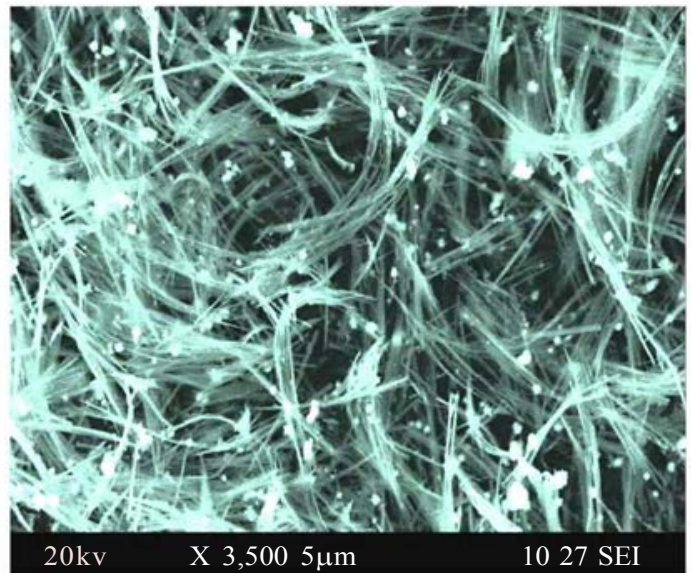

Fig. 4: TEM images of (a) A-TNTs (b) B-TNTs

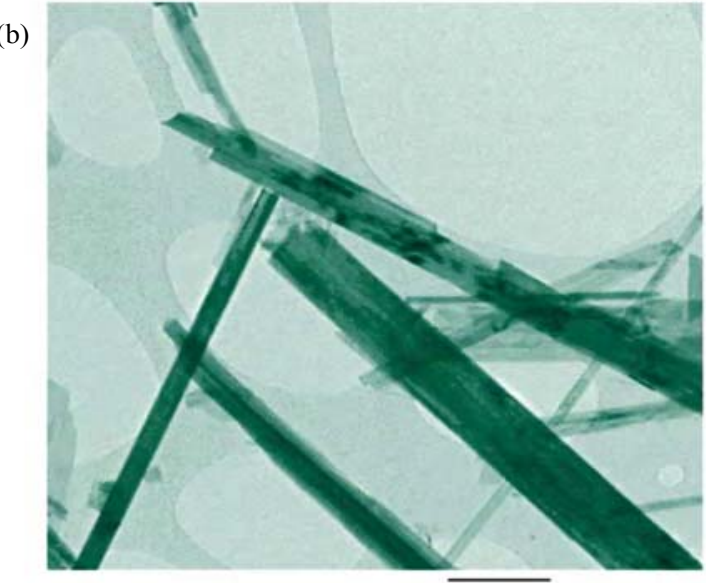


Table 2: EDX analysis of doped and undoped TNTs

\begin{tabular}{|c|c|c|c|c|c|}
\hline TNTs Desigination & $\mathrm{O}(\%)$ & $\mathrm{Ti}(\%)$ & $\mathrm{Na}(\%)$ & $\mathrm{Fe}(\%)$ & $\mathrm{Cr}(\%)$ \\
\hline A-TNTs & 21.31 & 77.87 & 0.82 & - & - \\
\hline B-TNTs & 20.01 & 70.29 & 0.14 & 4.36 & 5.2 \\
\hline
\end{tabular}

$F(R)=(1-R)^{2} / 2 R$

Where İR'I is the absolute value of reflectance. The energy bandgap of the doped and Fe/Cr doped TNTs were calculated from their diffuse-reflectance spectra by plotting the square of the Kubelka-Munk function $\mathrm{F}(\mathrm{R})^{2}$ vs energy in electron volts. The linear part of the curve was extrapolated to $F(R)^{2}=0$ to get the direct energy bandgap. The optical band gap of all samples was determined by the above method.

Fig. 5 shows the comparative the UV-vis diffuse reflectance spectrum of A-TNTs, B-TNTs taken at room temperature in the range of $200-800 \mathrm{~nm}$. It is clear from Fig. 4 that doping transition metals into titanium dioxide nanotubes tune the optical band gap in comparison to undoped TNTs, thus shifting UV absorption to visiblelight absorption and band gap narrowing. The band gap is reduced with doping of TNTs with Fe and $\mathrm{Cr}$ (Quan et al., 2005; Xu et al., 2005a; Kochkar et al., 2009) which was determined by K-Munk function. The narrower band gap, the more easily an electron is excited from the valence band to the conduction band. The differences in the band gap and light absorption property lead to different photocatalytic behavior. The
UV-vis diffuse reflectance spectra are in good agreement with the observed photocatalytic activity (Kubo et al., 2006).

Fig. $6 \mathrm{a}$ and $\mathrm{b}$ show the bandgap calculation of ATNTs, B-TNTs from K-Munk function, respectively. The direct band gap energies estimated from the intercept of the tangents to the plots are $3.25 \mathrm{eV}$ and $1.85 \mathrm{eV}$ for the samples A-TNTs and B-TNTs, respectively. The decrease in band gap energy of $\mathrm{B}$ TNTs can be ascribed to $\mathrm{Fe} / \mathrm{Cr}$ atom incorporated into the lattice of titanium dioxide. Thus, it induces a larger red-shift and bandgap narrowing thus changing the crystalline and electronic structures (Xu et al., 2005a; Kochkar et al., 2009).

It was also found, that constitution of the titanium dioxide nanotubes (doped and undoped $\mathrm{TiO}_{2}$ ) also plays an important role in its photoactivity This change in energy bandgap is directly reflected in the photocatlytic activity studies, where appreciable change is observed due doping (Dmitry et al., 2005; Xu et al., 2005b).

$\mathrm{TiO}_{2}$ nanoparticles were transformed to TNTs after hydrothermal treatment. However, the exact mechanism of formation and the morphology of TNTs is still

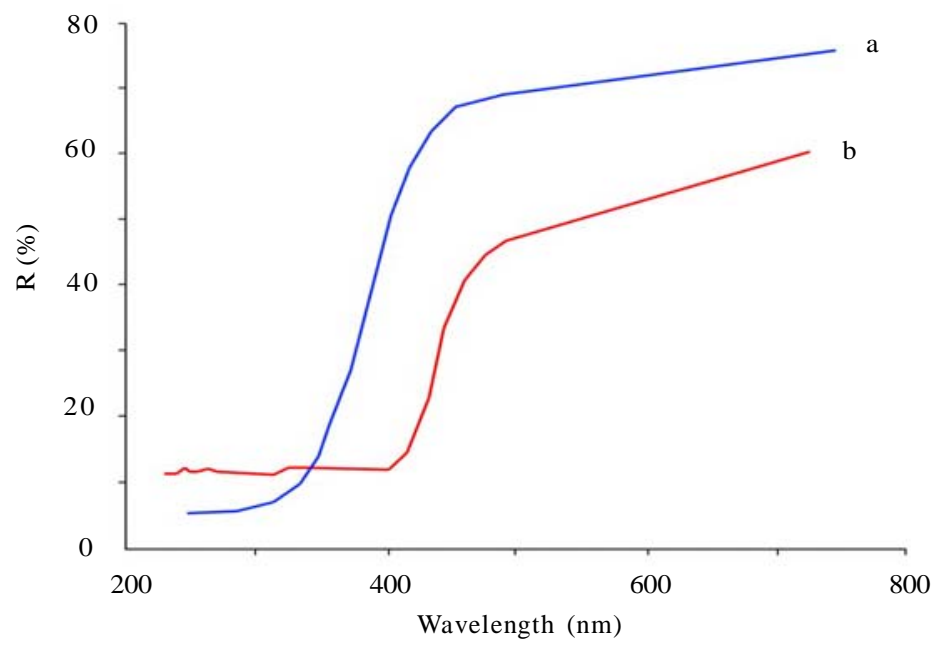

Fig. 5: UV-vis diffuse reflectance spectra of (a) A-TNTs and (b) B-TNTs 
subject of debate. There are few reports about the growth mechanism of titanium dioxide (Kubo et al., 2006). Kasuga et. al. (1998) proposed that TNTs are formed during the acid washing step (Chen et al., 2002a; Kubo et al., 2006). The nanotubes obtained after hydrothermal synthesis, showing very clear morphology and well defined structure. The nanotubes grow extensively with the length of several hundred nanometers with smooth tubular surfaces (Zhang et al., 2004).

From the results of present work and previous reports we proposed a plausible mechanism of formation of nanotubes. Certain Ti-O-Ti building units in titanium dioxide are broken down via alkali hydrothermal treatment of titanium dioxide with $\mathrm{NaOH}$, resulting in the formation of new co-ordination i.e.,Ti$\mathrm{O}-\mathrm{Na}$ and $\mathrm{Ti}-\mathrm{OH}$, lateron. Some of $\mathrm{Na}^{+}$ions were expected to be exchanged with $\mathrm{H}^{+}$ions during acid treatment and at this stage titanate sheets were exfoliated into layered nanosheets which subsequently scroll and form nanotubes at high temperature and high pressure to avoid electrostatic repulsions within the charges and to minimize the steric repulsions between the sheet edges while the tubular morphology is retained (Chen et al., 2002b; Liu et al., 2002; Zhang et al., 2004). These $\mathrm{H}^{+}$ions were then exchanged with $\mathrm{Fe}^{3+} / \mathrm{Cr}^{3+}$ while reaction of titanium dioxide nanotubes with their respective salts to obtain doped titanium dioxide nanotubes (Zhang et al., 2004).

To study the surface composition and chemical states of the synthesized nanotubes, the doped and undoped TNTs (A-TNTs and B-TNTs) were also analyzed by XPS and its XPS spectra are shown in Fig. 7a and b. The individual peaks of O1s at $529.5 \mathrm{eV}$ and Ti 2p at 458.2 and $463.9 \mathrm{eV}$ (Liu et al., 2002; Huogen et al., 2007) can be clearly seen in the highresolution spectra (Fig. 7a and b), which mean that chemical state of the sample is $\mathrm{Ti}^{4+}$ bonded with oxygen $\left(\mathrm{Ti}^{4+} \mathrm{O}\right)$, which has been modified by iron and chromium doping. The binding energy of the $\mathrm{Ti}$ $2 \mathrm{p} 3 / 2$ and $2 \mathrm{p} 1 / 2$ band in case of doped $\mathrm{Cr}_{2} \mathrm{O}_{3} / \mathrm{Fe}_{2} \mathrm{O}_{3}-$ $\mathrm{TiO}_{2}$ samples was found to be higher than that of the pure $\mathrm{TiO}_{2}$. This shift towards higher side, could be attributed to an atomic dispersion of iron and chromium on $\mathrm{TiO}_{2}$, and the formation of a $\mathrm{Ti}-\mathrm{O}-\mathrm{Cr} /$ Ti-O-Fe bond (Chen et al., 2002b; Kim et al., 2006; Huogen et al., 2007).

\section{Photocatalytic degradation of phenol}

The $\mathrm{TiO}_{2}$ anatase is the main catalyst used in the contaminants photodegradation. However, the photocatalytic activity of $\mathrm{TiO}_{2}$ nanotubes has been little explored in the literature (Kim et al., 2006; Huogen et al., 2007). Thus, a detailed study of the photocatalytic properties of $\mathrm{TiO}_{2}$ nanotubes is needed. In this study, photocatalytic activity of the mesoporous titanium dioxide nanotube composites was evaluated by photocatalytic phenol degradation, the results are shown in Fig. 8, under infra-red (IR) and visible light illumination. The degradation kinetics was computed after every $30 \mathrm{~min}$ by the change in phenol concentration ( $1 \mathrm{~g} / \mathrm{L}$ ) employing UV-visible spectrometry as a function of irradiation time. The
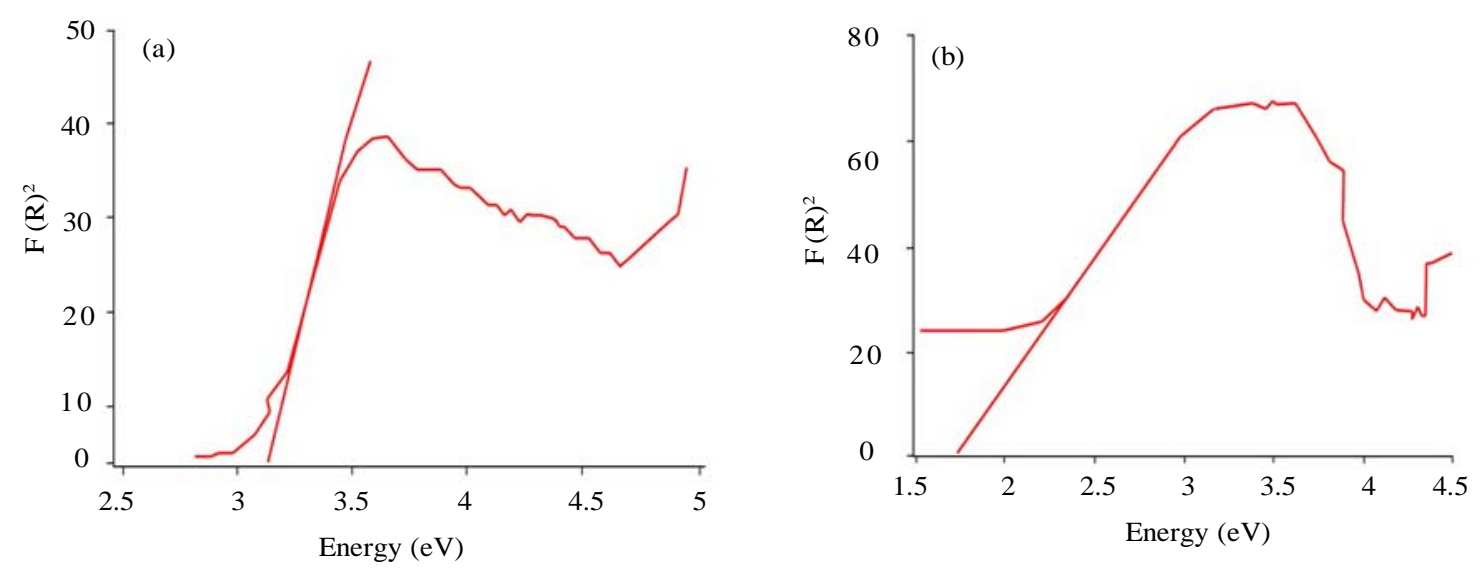

Fig. 6: Bandgap measurements of (a) A-TNTs (b) B-TNTs 
phenol conversion is estimated using the following equation (Hou et al., 2007).

Phenol Conversion $(\%)=[\text { Phenol }]_{0}-[\text { Phenol }]_{\mathrm{t}}$ $/[\text { Phenol }]_{0} \times 100$

Where $[\text { Phenol }]_{0}$ is the initial concentration of phenol, [Phenol $]_{\mathrm{t}}$ is the concentration of phenol after time " $t$ ". The study of Fig. 8 indicates that Fe/Cr doped nanotubes decompose phenol more rapidly than does titanium dioxide nanotubes alone under UV light. Similar trend is observed in case of IR irradiation.

The photocatalytic decomposition of phenol is remarkably accelerated by the doped-TNTs photocatalysts prepared in the present work. This may be due to the inhibition of a spontaneous recombination between the hole and electron occurring on the surface of excited TNTs (Xu et al., 2005b) by the metals deposited on the TNTs surface. The photodegradation efficiency of titanate nanotubes is $35 \% \pm 3$, while after doping the efficiency increased to $75 \% \pm 3$ under IR irradiation. Metal ion doping influence the photoactivity of $\mathrm{TiO}_{2}$ by electron or hole traps. Where the trap causes the formation of some active species that benefit degradation of phenol, here the electron scavenger effect of $\mathrm{Cr}^{3+} / \mathrm{Fe}^{3+}$ prevents the recombination of electron and hole pairs thus resulting in increase of the efficiency of photodegradation process (Kim et al., 2006; Hou et al., 2007). In Fe/Cr- doped samples, electrons are either directly trapped at $\mathrm{Ti}$ (IV) surface sites (form $\mathrm{Ti}^{3+}$ ) or in deeper $\mathrm{Fe}$ (III)/ $\mathrm{Cr}$ (III) sites (form $\mathrm{Cr}^{2+} / \mathrm{Fe}^{2+}$ ). In this case, the trapped electron can be easily transferred from $\mathrm{Cr}^{2+} / \mathrm{Fe}^{2+}$ to a neighboring surface $\mathrm{Ti}^{4+}$ because of the proximity of the energy levels (Dmitry et al., 2005; Huogen et al., 2007; Hou et al., 2007). As a result, the photocatalytic activity is improved after the doping. The proposed mechanism of process is presented in Fig. 9. The titanium dioxide nanotubes fabricated through hydrothermal method showed large surface are and high reactivity. Moreover, doping of titanium dioxide nanotubes with transition metals like chromium and iron, resulted in the enhanced photocatalytic properties because of the decrease bandgap. These materials provide sufficient negative and positive redox potential, which accelerate the degradation process.

\section{Conversion of $\mathrm{CO}_{2}+\mathrm{H}_{2} \mathrm{O}$ by solar energy}

The conversion of $\mathrm{CO}_{2}+\mathrm{H}_{2} \mathrm{O}$ into alcohol by the synthesized undoped and doped titanium dioxide nanotubes was evaluated under UV and IR radiation, respectively and presented in Fig. 10a and b. The comparative stdudy of Fig. 10a and b reveals that the doped TNTs show considerably higher conversion of $\mathrm{CO}_{2}+\mathrm{H}_{2} \mathrm{O}$ in comparison with the undoped nanotubes under both IR and UV irradiation. $\mathrm{CO}_{2}$ conversion is calculated using the following formula (Huogen et al., 2007).
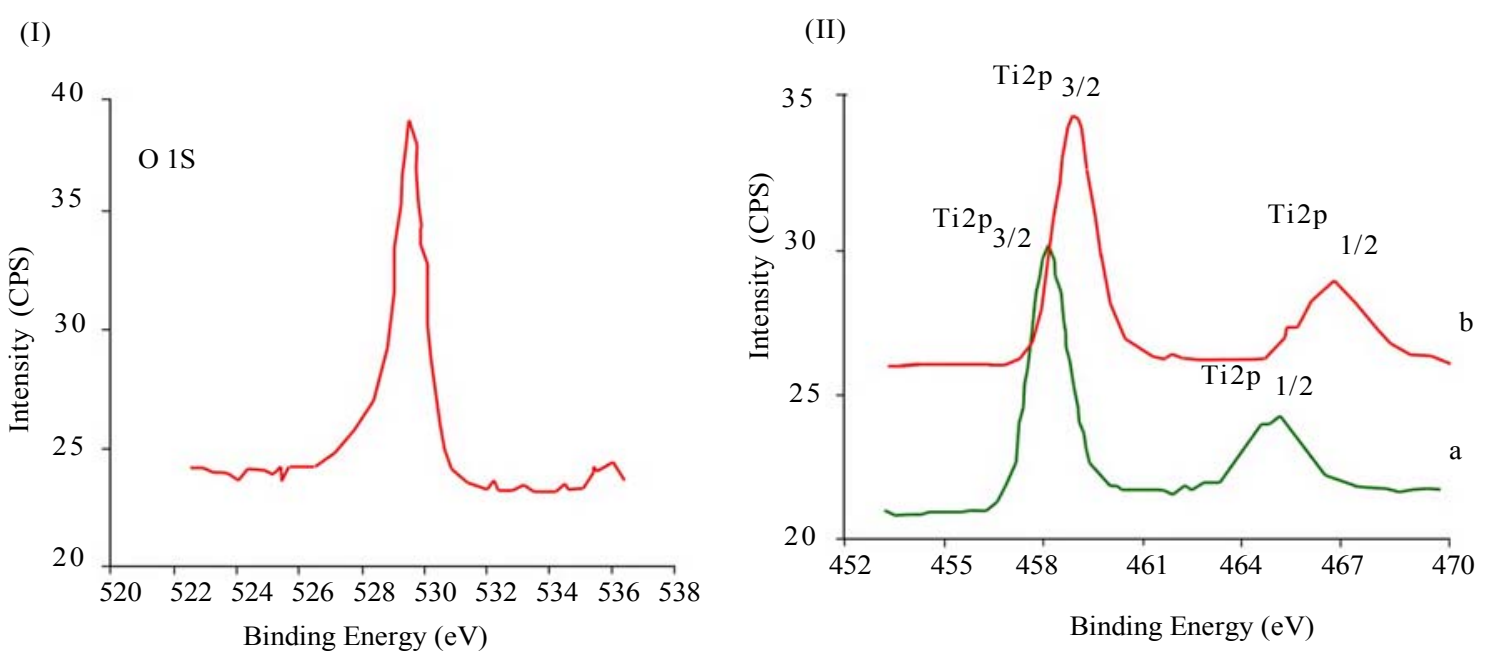

Fig. 7: XPS spectra of (I) O and (II) a) A-TNTs b) B-TNT 
$\mathrm{CO}_{2}$ conversion $(\%)=\left[\mathrm{CO}_{2}\right]_{\mathrm{t}} /\left[\mathrm{CH}_{3} \mathrm{OH}\right]_{\mathrm{t}} \times 100$

Where, $\left[\mathrm{CO}_{2}\right]_{\mathrm{t}}=\mathrm{CO}_{2}$ conversion at time $t$. $\left[\mathrm{CH}_{3} \mathrm{OH}\right]_{\mathrm{t}}=$ Formation of $\mathrm{CH}_{3} \mathrm{OH}$ at time t. When titanium dioxide absorbs UV radiation from sunlight or illuminated light source, it will produce pair of electrons and holes. The electrons of valence band are excited when illuminated by light. The excess energy of this excited electro promoted the electron to the conduction band of titanium dioxide therefore producing the negative electron (e-) and positive hole $(\mathrm{h}+)$ pair within the catalyst (Xu et al., 2005a). A portion of this photoexcited electron-hole pairs diffuse to the surface of the catalytic particle (electron hole pairs are trapped at the surface) and take part in the chemical reaction with the adsorbed donor (D) or acceptor (A) molecules (Syoufian et al., 2007). The positive holes can oxidize donor molecules i.e., break apart water molecules to form hydrogen gas and hydroxyl radicals which combines with carbon to form alcohol, whereas the negative electrons can reduce appropriate electron acceptor molecules i.e., react with oxygen molecules

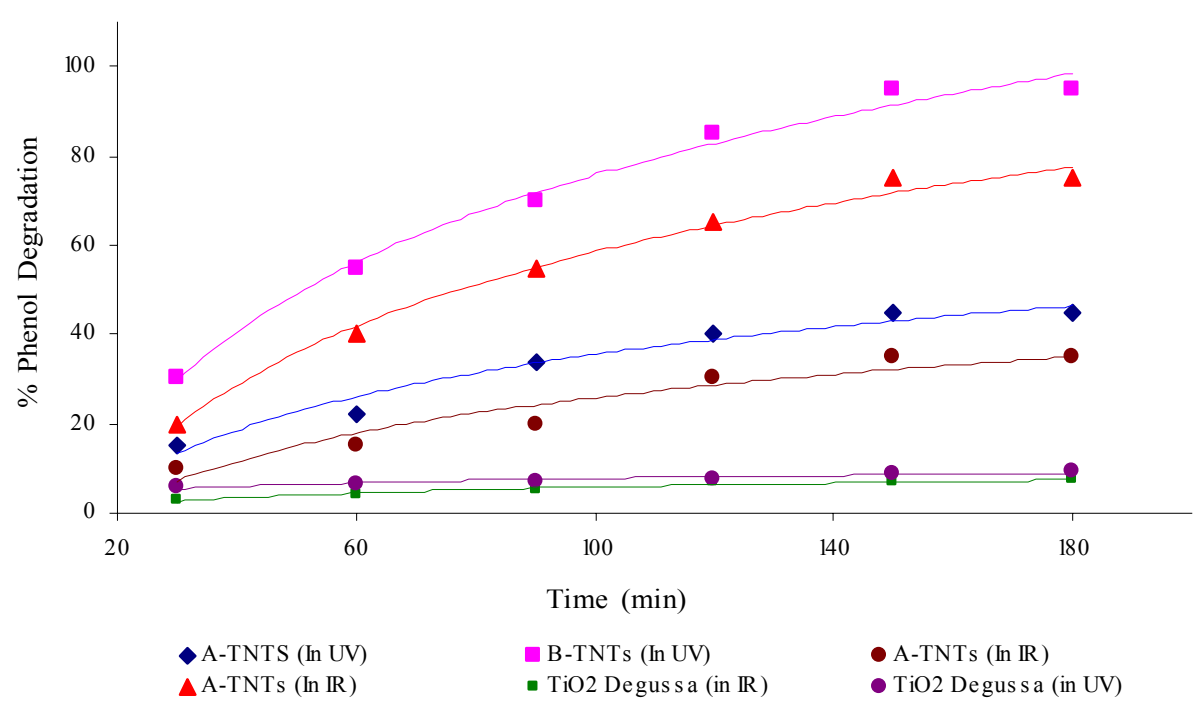

Fig. 8: Photocatalytic degradation of Phenol by $0.01 \mathrm{gm}$ of TiO2 degussa, A-TNTs and B-TNTs under UV and IR irradiation at room temperature with different time rates

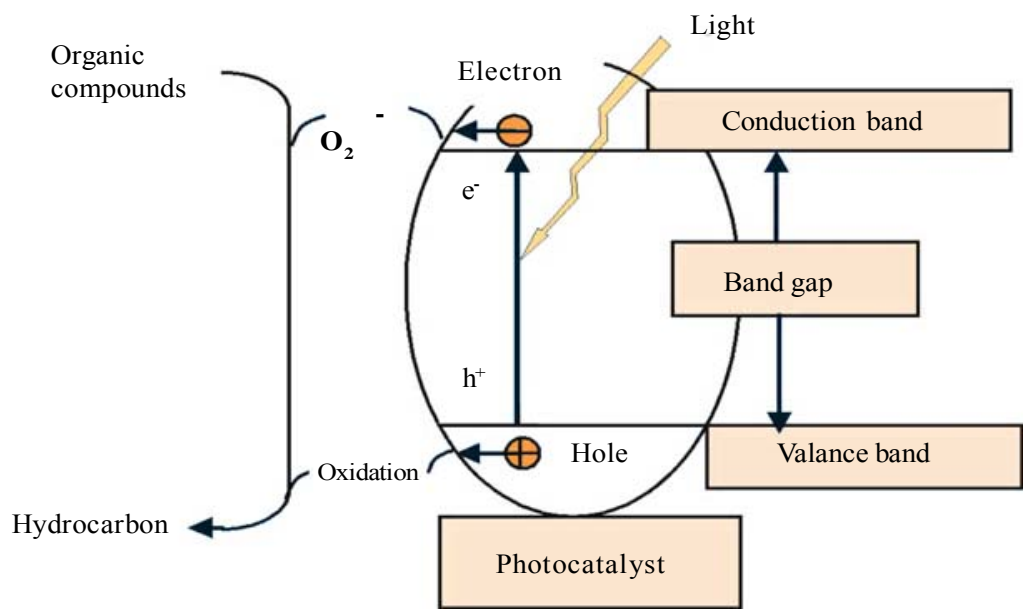

Fig. 9: The proposed photocatalytic activity mechanism 

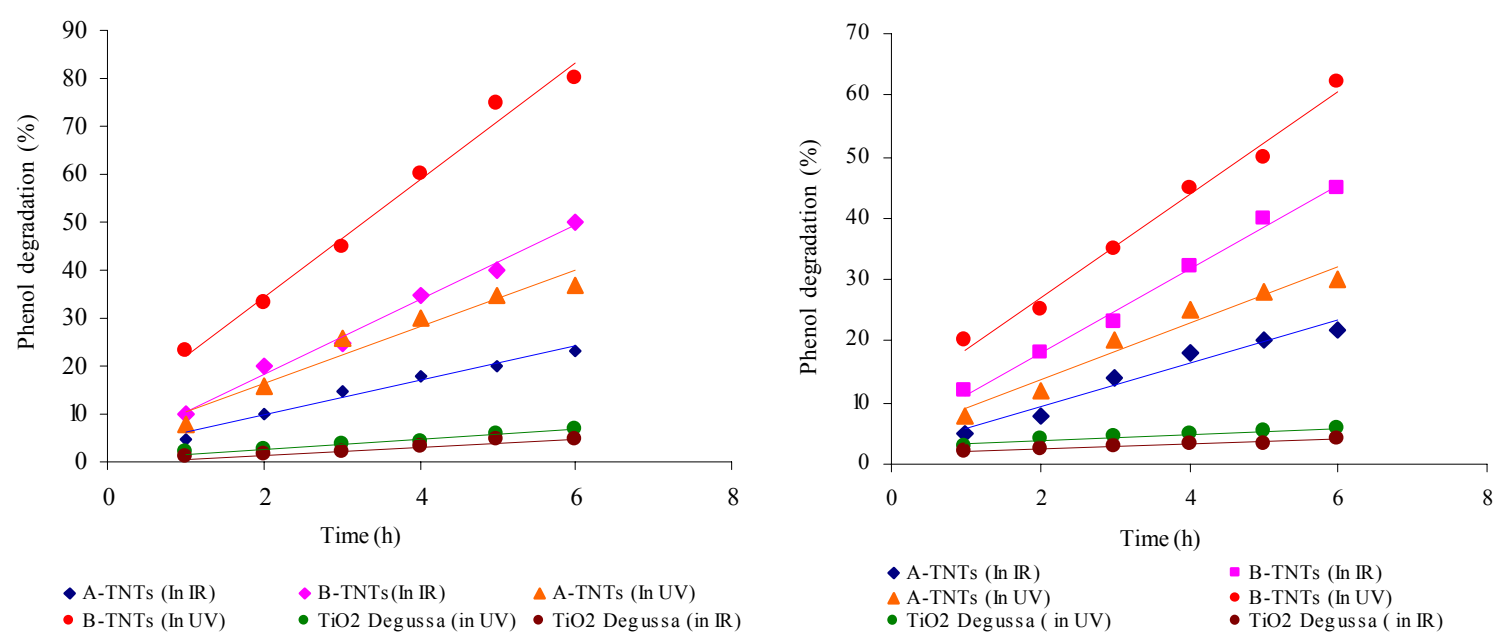

Fig. 10 (a and b): \% production (a) ethanol (b) methanol by 0.01 gm of A-TNTs and B-TNTs under UV and IR irradiation at room temperature with different time rates

to form super oxide anion (Fig. 9), illuminated $\mathrm{TiO}_{2}$ photocatalysts can decompose and mineralize organic compounds by participating in a series of oxidation reactions leading to carbon dioxide to harmless substance that can be released to the environment (Xu et al., 2005b; Syoufian et al., 2007). This cycle continue when the sun light is available. The energy needed to activate TNTs is $3.2 \mathrm{eV}$ or more, which nearly corresponds to UV radiation wavelength of $380 \mathrm{~nm}$ or less. This makes it possible to use the sun as illumination source, since about 4-6\% of the solar energy that reaches the earth's surface is less than $400 \mathrm{~nm}$. This process is referred to as heterogeneous photocatalysis or more specifically, photocatalysis oxidation (PCO). The energy difference between valence band and conduction band is referred as band gap (Jin and Shiraishi, 2004).

Hereby it is proposed that the $\mathrm{Fe}^{3+}$ and $\mathrm{Cr}^{3+}$ presence inside the lattice of $\mathrm{TiO}_{2}$ nanotubes, induces a delocalized electron density within $\mathrm{CO}_{2}+\mathrm{H}_{2} \mathrm{O}$ structure that promotes the adsorption of the molecule on to the surface. This effect could support the reaction pathway to the mineralization on to $\mathrm{TiO}_{2}$ surface with consequent yields of intermediate species and creates extra energy due to presence of dopand which results in the breakage of $\mathrm{H}-\mathrm{OH}$ and $\mathrm{C}-\mathrm{O}$ bonds. It is speculated that molecules can adsorb through the formation of $\mathrm{Ti}^{+4}$ (surface) $\pi$ electron or $\mathrm{OH}$ (surface) $\pi$ electron type complex and latter is related to the production of products. For both type of adsorption, the nature of promoter related to their withdrawing or donor capacity played a major role. All types of Lewis and BronstedLowry sites are generally present at various extents on the lattice of $\mathrm{TiO}_{2}$ nanotubes (Jin and Shiraishi, 2004; Syoufian et al., 2007). In the $\mathrm{CO}_{2}+\mathrm{H}_{2} \mathrm{O}$ conversion to alcohol, two important species are involved in the process: $\mathrm{H}^{+}$(hydrogen radical) and $\mathrm{CO}_{2}^{-}$ (carbon dioxide anion radical). The incident photons are observed by $\mathrm{TiO} 2$ and photoexcited electron (e-) and positive hole $(\mathrm{h}+)$ are formed in the catalyst due to presence of $\mathrm{Fe}^{3+}$ and $\mathrm{Cr}^{3+}$ (i.e., by a charge transfer to the excited site of Fe-Cr-Ti-O). Furthermore, the photoexcited electrons and holes in the lattice are separated and trapped by appropriate sites of $\mathrm{Fe}-\mathrm{TiO}_{2}$ and $\mathrm{Cr}-\mathrm{TiO}_{2}$. The whole react with $\mathrm{H}_{2} \mathrm{O}$ adsorbed on the surface of the catalyst giving rise to oxygen and $\mathrm{H}^{+}$. The $\mathrm{H}^{+}$ions interact with excited electrons, resulting the formation of $\mathrm{H}^{+}$radical. This is enhanced by the presence of multiple active sites of $\mathrm{Fe}$ and $\mathrm{Cr}$ coupled with $\mathrm{TiO}_{2}$. The interaction of all these species results in the formation of alcohol. The role that which types of sites are responsible for the photocataytic reaction is difficult to assess. This study is under way at present and will be published later.

\section{CONCLUSION}

In this work, mesoporous nanotubes by hydrothermal treatment in aqueous sodium hydroxide solution have been synthesized. Direct hydrothermal synthesis method is easy and efficient to synthesize 
pure and titanate nanotubes doped with transition metals. Using methanol and sodium hydroxide mixture, the considerable decrease in reaction time and reaction temperature was achieved.

High aspect ratio of $\mathrm{TiO}_{2}$ undoped and doped $\mathrm{TiO}_{2}$ nanotubes of homogenous length were synthesized. XRD result suggests that cryatallanity enhanced after annealing, while the band gap and BET surface area increased after doping. TNTs revealed a larger specific surface area and a higher pore volume than that of nanoparticles, leading to an obvious enhancement of the photocatalytic activity for the photocatalytic degradation of pollutants ( $\mathrm{CO}_{2}$ and phenol) under UV and IR irradiation. Doping of metals, such as iron and chromium on the surface of TNTs resulted in enhanced rate of the photocatalytic decomposition of organic compounds in aqueous solutions.

In conclusion, $\mathrm{TiO}_{2}$ nanotubes are found to be feasible and attractive to be used in further investigation of $\mathrm{CO}_{2}$ reduction for $\mathrm{CO}_{2}$ environment management. The first three conclusion points exclusively explained the scientific achievement. The low temperature, low pressure with homogeneous tube length, very good aspect ratio and high surface area with band gap in the UV range will directly affect the economics of all the process.

\section{REFERENCES}

Chen, Y. S.; Crittenden, J. C.; Hackney, S.; Sutter, L.; Hand, D. W., (2005). Preparation of a novel TiO2-based p-n junction nanotube photocatalyst. Environ. Sci. Tech., 39 (5), 1201-1208 (8 pages).

Chen, Q. ; Zhou, W.; Du, G .; Peng, L-M., (2002a). Titanate nanotubes made via single alkali treatment. Adv. Mat., 14 (17), 1208-1211 (4 pages).

Chen, Q.; Du, G. H.; Zhang, S.; Peng, L. M., (2002b). The structure of trititanate nanotubes. Acta Cryst., B, 58 (4), 587-593 ( 7 pages).

Chien, M. K.; Shih, L. H., (2007). An empirical study of the implementation of green supply chain management practices in the electrical and electronic industry and their relation to organizational performances. Int. J. Environ. Sci. Tech., 4 (3), 383-394 (12 pages).

Diebold, U., (2002). Structure and properties of $\mathrm{TiO}_{2}$ surfaces: A brief review. Appl. Phys. A., 76 (5), 1-7 (7 pages).

Dmitry, V.; Alexei, A. L.; Pawel, K.; Jens, M. F.; Frank, C. W.; Walsh, C., (2005). TiO2 nanotube-supported ruthenium (III) hydrated oxide:A highly active catalyst for selective oxidation of alcohols by oxygen. J. Catal., 235 (1), 10-17 (8 pages).

Fujishima, A.; Zhang, X.; Chimie, C. R., (2006). Titanium dioxide photocatalysis: present situation and future approaches. Comptes Rendus Chimie, 9 (5-6), 750-760 (11 pages).
Goyal, P.; Sharma, P.; Srivastava, S.; Srivastava, M. M., (2008). Saraca indica leaf powder for decontamination of $\mathrm{Pb}$ : removal, recovery, adsorbent characterization and equilibrium modeling. Int. J. Environ. Sci. Tech., 5 (1), $27-$ 34 (8 pages).

Hou, L. R.; Yuan, C. Z.; Peng, Y., (2007). Synthesis and photocatalytic property of $\mathrm{SnO}_{2} / \mathrm{TiO}_{2}$ nanotubes composites. J. Hazard. Mater. B., 139 (2), 310-315 (6 pages).

Huogen, Y.; Jiaguo, Y.; Chenga, B.; Lin, J., (2007). Synthesis, characterization and photocatalytic activity of mesoporous titania nanorod/titanate nanotube composites. J. Hazard. Mater., 147 (1-2), 581-587 (7 pages).

Hussain, S. T.; Khan, K.; Hussain, R., (2009). Size control synthesis of sulfur doped titanium dioxide (anatase) nanoparticles, its optical property and its photo catalytic reactivity for $\mathrm{CO} 2+\mathrm{H} 2 \mathrm{O}$ conversion and phenol degradation. J. Nat. Gas Chem., 18, 383-391 (9 pages).

Ismael, C.; Freitas, J.; Longoa, C.; Aurelio, M.; Winnischofer, H.; Nogueira, A., (2007). Dye-sensitized solar cells based on TiO2 nanotubes and a solid-state electrolyte. J. Photochem. Photo. A: Chem., 189 (2-3), 153-160 (8 pages).

Jin, S.; Shiraishi, F., (2004). Photocatalytic activities enhanced for decompositions of organic compounds over metalphotodepositing titanium dioxide. Chem. Eng. J., 97 (2-3), 203-211 (9 pages).

Kasuga, T.; Hiramatsu, M.; Hoson, A.; Sekino, T.; Niihara, K., (1998). Formation of titanium oxide nanotube. Lang. 14 (12), 3160-3163 (4 pages).

Khanfekr, A.; Arzani, K.; Nemati, A.; Hosseini, M., (2009). Production of perovskite catalysts on ceramic monoliths with nanoparticles for dual fuel system automobiles. Int. J. Environ. Sci. Tech., 6 (1), 105-112 (8 pages).

Khan, M. A.; Jung, H. T.; Yang, O. B., (2006). Synthesis and characterization of ultrahigh crystalline $\mathrm{TiO} 2$ nanotubes. J. Phys. Chem., B 110 (13), 6626-6630 (5 pages).

Kim, J. C. l.; Choi, J.; Bok Y; Jung, L.; Hong, H.; Yang, J. W.; Lee, W. I.; Hur, N. H., (2006). Enhanced photocatalytic activity in composites of $\mathrm{TiO} 2$ nanotubes and $\mathrm{CdS}$ nanoparticles. Chem. Commun., 5 (48), 5024-5026 (3 pages).

Kochkar, H.; Turki, A.; Bergaoui, L.; Berhault, G.; Ghorbel, A., (2009). Study of Pd(II) adsorption over titanate nanotubes of different diameters. J. Coll. Interf. Sci., 331 (1), 27-31 (5 pages).

Kuang, D.; Brillet, J.; Chen, P.; Takata, M.; Uchida, S.; Miura, H., (2008). Application of highly ordered TiO2 nanotube arrays in flexible dye- sensitized solar cells. ACS Nano, 2 (1), 1113-1116 (4 pages).

Kubo, T.; Yamasaki, Y.; Honma, T.; Umesaki, N.; Nakahira, A., (2006). Synthesis of nanotubular H-Ti-O by hydrothermal process and its structural evaluation. Materials, 2, 1-6 (6 pages).

Liang, H.; Li, X., (2009). Effects of structure of anodic $\mathrm{TiO}_{2}$ nanotube arrays on photocatalytic activity for the degradation of 2, 3-dichlorophenol in aqueous solution. J. Hazard. Mat., 162 (2-3), 1415-1422 (8 pages).

Liu, S. M.; Gan, L. M.; Liu, L. H.; Zhang, W. D.; Zeng, H. C., (2002). Synthesis of Single-Crystalline TiO2 Nanotubes. Chem. Mater., 14 (3), 1391-1397 (7 pages).

Macak, J. M.; Tsuchiya, H.; Ghicov, A.; Yasuda, K.; Hahn, R.; Bauer, S.; Schmuki, P., (2007). TiO nanotubes: Self- 
organized electrochemical formation, properties and applications. Curr. Opin. Solid. Mat. Sci., 11 (1-2), 3-18 (16 pages).

Mohapatra, S. K.; Misra, M.; Mahajan, V. K.; Raja, K. S., (2007). A novel method for the synthesis of titania nanotubes using sonoelectrochemical method and its application for photoelectrochemical splitting of water. J. Catal., 246 (2), 362-369 (7 pages).

Qamar, M.; Yoo, C. R.; Oh, H. J.; Lee, N. H.; Park, K.; Kim, D. H.; Lee, K. S.; Lee, W. J.; Kim, S. J., (2008). Preparation and photocatalytic activity of nanotubes obtained from titanium dioxide. Catal. Today, 131 (1-4), 3-14 (12 pages).

Quan, X.; Yang, S. G.; Ruan, X. L.; Zhao, H. M., (2005). Preparation of titania nanotubes and their environmental applications as electrode. Environ. Sci. Tech., 39 (10), 37703775 (6 pages).

Rafati, L.; Mahvi, A. H.; Asgari, A. R.; Hosseini, S. S., (2010). Removal of chromium (VI) from aqueous solutions using Lewatit FO36 nano ion-exchange resin. Int. J. Environ. Sci. Tech., 7 (1), 147-156 (10 pages).

Samarghandi, M. R.; Nouri, J.; Mesdaghinia, A. R.; Mahvi, A. H.; Nasseri, S.; Vaezi, F., (2007). Efficiency removal of phenol, lead and cadmium by means of UV/ TiO2/ $\mathrm{H} 2 \mathrm{O} 2$ processes. Int. J. Environ. Sci. Tech., 4 (1), 19-26 (8 pages).

Sekabira, K.; Oryem Origa, H.; Basamba, T. A.; Mutumba, G.; Kakudidi, E., (2010). Heavy metal assessment and water quality values in urban stream and rain water. Int. J. Environ. Sci. Tech., 7 (4), 759-770 (12 pages).

Sun, X.; Li, Y., (2003). Synthesis and characterization of ion exchangeable titania nanatubes. Chem. Eur. J., 9 (10), 22292238 (10 pages).
Syoufian, A.; Oktaviano, H. S.; Nakashima, K., (2007). Photodecomposition of methylene blue as a target molecule. Catal. Commun., 8 (5), 755-759 (5 pages).

Wang, W.; Varghese, O. K.; Paulose, M.; Grimes, C. A., (2004). A study on the growth and structure of titania nanotubes. J. Mat. Res., 19 (2), 417-422 (6 pages).

Xu, J. C.; Mei, L.; Yong, X. G.; Li, H. L., (2005a). Zinc ions surface-doped titanium dioxide nanotubes and its photocatalysis activity for degradation of methyl orange in water. J. Mol. Catal. A: Chem., 226 (1), 123-137 (15 pages).

Xu, M. W.; Bao, S. J.; Zhang, X. G., (2005b). Enhanced photocatalytic activity of magnetic $\mathrm{TiO} 2$ photocatalyst by silver deposition. Mater. Lett., 59 (17), 2194-2198 (5 pages).

Yury, V.; Kirill, A.; Anton, I.; Alexei, V.; Johan, F.; Oleg, I.; Bulat, R.; Churagulov, O.; Yoshimura, M., (2000). Hydrothermal synthesis and characterization of nanorods of various titanates and titanium dioxide. J. Phys. Chem. B., 110 (9), 4030-4038 (9 pages).

Zhuang, H.; Lin, C.; Lai, Y.; Sun, L.; Li, J., (2007). Some Critical Structure Factors of Titanium Oxide Nanotube Array in Its Photocatalytic Activity. Environ. Sci. Tech., 41 (13), 4735-4740 (6 pages).

Zhu, T.; Li, J.; Jin, Y. Q.; Liang, Y. H.; Ma, G. D., (2009). Gaseous phase benzene decomposition by non-thermal plasma coupled with nano titania catalyst. Int. J. Environ. Sci. Tech., 6 (1), 141-148 (8 pages).

Zhuang, M.; Jin, Z. S.; Yung, J. J.; Zhang, Z. J., (2004). Effect of annealing temperature on morphology, structure and photocatalytic behavior of nanotubed $\mathrm{H}_{2} \mathrm{Ti}_{2} \mathrm{O}_{4}(\mathrm{OH})_{2}$. J. Molec. Catal. A: Chem., 217 (1-2), 203-210 (8 pages).

\section{AUTHOR (S) BIOSKETCHES}

Hussain, S. T., Ph.D., Director, Nanoscience and Catalysis Division, National Center for Physics, Quaid-i-Azam University Islamabad, Pakistan. Email: dr_tajammul@yahoo.ca

Siddiqa, A., Research Scientist, Nanoscience and Catalysis Division, National Center for Physics, Quaid-i-Azam University Islamabad, Pakistan. Email: Asima_siddiqa@yahoo.com 